\title{
Território, intersetorialidade e escalas: requisitos para a efetividade dos Objetivos de Desenvolvimento Sustentável
}

\author{
Territory, intersectoriality and stages: requirements \\ for the effectiveness of the sustainable development goals
}

Edmundo Gallo ${ }^{1}$

Andréia Faraoni Freitas Setti ${ }^{2}$

${ }^{1}$ Escola Nacional de Saúde Pública, Fundação Oswaldo Cruz (Fiocruz). R. Leopoldo Bulhões 1480, Manguinhos. 21041-210 Rio de Janeiro RJ Brasil.gallo@fiocruz.br ${ }^{2}$ Observatório de Territórios Sustentáveis e Saudáveis, Fiocruz.

\begin{abstract}
The post-2015 development agenda highlights the incorporation of sustainability in approaches developed and/or applied to distinct fields of knowledge and action and the demonstration of the effectiveness of experiences of sustainable and healthy territories. This process results from the confrontation of different viewpoints which seek to address social production vis-à-vis their project, with the possibility of updating the hegemonic mode of production and consumption or the emergence of counter-hegemonic rationales. Health, as one of the SDGs, has the challenge of imposing an intersectorial agenda that addresses its social determinants, in a process of participative governance able to build a hierarchy of priorities based on the needs of the territory and build techno-political solutions based on the ecology of knowledge, constituting a strategic-situational and communicative management process. The consistency in formulation of the agenda and potential challenges to its implementation are analyzed, considering its intersectoriality, its strategic governance and management, and especially an assessment of its effectiveness. Moreover, it tests the evaluative tools used and their ability to analyze the consistency in the formulation of the agenda.
\end{abstract}

Key words Post-2015 agenda, Sustainable development goals, Evaluation of effectiveness
Resumo A Agenda do Desenvolvimento pós2015 destaca a incorporação da sustentabilidade nas abordagens desenvolvidas e/ou aplicadas aos diferentes campos de conhecimento e ação e a demonstração da efetividade de experiências de territórios sustentáveis e saudáveis. Este processo resulta do enfrentamento de distintas visões que buscam dirigir a produção social no sentido de seu Projeto, com possibilidades de atualização do modo de produção e consumo hegemônicos ou da emergência de racionalidades contra-hegemônicas. A saúde, um dos Objetivos de Desenvolvimento Sustentável, tem o desafio de inserir-se em uma agenda intersetorial e interescalar que enfrente seus determinantes sociais em um processo de governança participativo, capaz de construir uma hierarquia de prioridades a partir das necessidades do território e de produzir soluções tecnopolíticas baseadas na ecologia de saberes, constituindo um processo de gestão estratégico situacional e comunicativo. Analisa-se a consistência da formulação da Agenda e potencialidades e desafios para a sua implantação, considerando a intersetorialidade e a interescalaridade, sua governança e gestão estratégica, em especial a avaliação de efetividade. Adicionalmente, testa as ferramentas avaliativas utilizadas em sua capacidade de analisar a consistência da formulação da Agenda.

Palavras-chave Agenda pós-2015, Objetivos de Desenvolvimento Sustentável, Avaliação de efetividade 


\section{Introdução}

O processo de definição dos Objetivos de Desenvolvimento Sustentável (ODS), intensificado após a realização da Rio+20, em Junho de 2012, vem colocando em destaque a importância de incorporar a dimensão da sustentabilidade nas abordagens que vêm sendo desenvolvidas e/ou aplicadas nos distintos campos de conhecimento e ação ${ }^{1}$.

No caso da saúde, isso é reafirmado nos resultados da Conferência Internacional de Saúde Urbana, da Conferência Mundial sobre Determinantes Sociais da Saúde e da própria Rio+20, que apontam como estratégica para o alcance da sustentabilidade a implantação e demonstração da efetividade de experiências teórico-práticas para a construção de territórios sustentáveis e saudáveis ${ }^{2-8}$.

Como processo histórico, a construção de territórios sustentáveis e saudáveis é resultado do enfrentamento de distintas visões que buscam dirigir a produção social do espaço e das territorialidades no sentido de seu Projeto, com possibilidades de atualização do modo de produção e consumo hegemônicos ou da emergência de racionalidades contra-hegemônicas.

Neste sentido, a saúde, como uma das dezessete áreas preliminarmente definidas como um dos ODS no processo de consulta global no documento do High-Level Panel of Eminent Persons on the Post-2015 Development Agenda: A New Global Partnership Eradicate Poverty and Transform Economies through Sustainable Development ${ }^{9}$, e no informe do Open Working Group Introduction and Proposed Goals and Targets on Sustainable Development for the Post 2015 Development Agenda $a^{10}$ - coloca para seus atores o desafio de inserir-se em uma agenda intersetorial (para além da saúde) e interescalar (do local ao global), capaz de impulsionar o enfrentamento dos seus determinantes sociais em territórios de diferentes escalas, o que dialoga estreitamente com o desenvolvimento sustentável ${ }^{11,12}$. Isto significa: 1) atualizar e aprender com a agenda dos Objetivos de Desenvolvimento do Milênio - ODM e articulá-la aos ODS; 2) definir um ODS para a área que seja universal, intersetorial, adaptável às realidades locais, mensurável e de fácil comunicação; 3) definir sua contribuição para os outros dezessseis temas propostos; e 4) implantar agendas territorializadas capazes de efetivar os ODS em uma perspectiva contra-hegemônica. Para que as agendas atendam a esses requisitos e para que seja demonstrada sua efetividade, sua implantação implica na constituição de arranjos intersetoriais e interescalares que envolvam os distintos atores em um processo de governança participativo, capaz de construir uma hierarquia de prioridades a partir das necessidades do território e de construir soluções tecnopolíticas baseadas na ecologia de saberes, constituindo um processo de gestão estratégico-situacional e comunicativo ${ }^{13}$. Isso demanda o monitoramento e a avaliação da efetividade dos ODS, de forma intersetorial e interescalar, como apontado na Declaração Política do Rio sobre Determinantes Sociais da Saúde, 2011, onde são considerados requisitos para fundamentar políticas e desenvolver a responsabilidade sobre os determinantes sociais, principalmente monitorando tendências das iniquidades em saúde e dos impactos das ações sobre elas, desenvolvendo pesquisas "sobre as relações entre os determinantes sociais da saúde e a equidade em saúde, com foco especial na avaliação da eficácia das intervenções"14 e promovendo sistemas de monitoramento apropriados e participativos. No mesmo sentido, a Declaração da Rio+20 "O futuro que queremos", no parágrafo 76 , alínea g, destaca a importância de promover capacidades em avaliação de pesquisas e tecnologias, buscando um constante diálogo voltado para a elaboração e avaliação de estratégias de desenvolvimento sustentável, promovendo el nexo entre la ciencia y las políticas mediante evaluaciones científicas inclusivas, transparentes y basadas en pruebas, así como el acceso a datos fiables, pertinentes y oportunos en las esferas relacionadas con las tres dimensiones del desarrollo sostenible ${ }^{1}$. Este artigo analisa a consistência da formulação da Agenda do Desenvolvimento pós-2015 em relação ao desenvolvimento sustentável e as potencialidades e os desafios para a sua implantação efetiva, considerando especialmente a intersetorialidade e a interescalaridade em seu rebatimento sobre o território, e sua expressão em agendas territorializadas, cuja governança e gestão estratégica, em especial a avaliação de efetividade, são destacadas. Adicionalmente, testa as ferramentas avaliativas utilizadas em sua capacidade de analisar a consistência da formulação da Agenda.

\section{Metodologia}

Elaborou-se revisão e análise crítica dos documentos produzidos pelo Painel de Alto Nível das Nações Unidas (High-Level Panel - HLP) ${ }^{9} e$ pelo Grupo de Trabalho Inter-governamental da ONU (UN Open Working Group on Sustainable 
Development) ${ }^{10}$ considerando-se, ainda, outros documentos do processo de definição da Agenda pós-2015: O futuro que queremos $(\text { Rio }+20)^{1}$; Health in the post-2015 Agenda (Botswana) ${ }^{15}$; e os informes das diversas consultas temáticas globais levadas a cabo pelas Nações Unidas ${ }^{16}$, tendo como base o informe "Saúde na Agenda do Desenvolvimento pós-2015”, elaborado pelo Grupo Técnico da Fiocruz sobre Desenvolvimento Sustentável e Saúde ${ }^{17}$. Tomou-se como referencial teórico as abordagens ecossistêmica e comunicativa do planejamento estratégico, que consideram a governança territorial participativa como elemento central para a factibilidade e viabilidade de estratégias de Desenvolvimento Sustentável e Promoção da Saúde. Para analisar a consistência da Agenda pós-2015, em relação ao desenvolvimento sustentável e saudável, utilizouse tecnologias de avaliação de efetividade que procuram integrar as dimensões teórico-práticas destas estratégias, considerando suas agendas de implementação: Agenda 21, Territórios/Cidades Saudáveis ${ }^{8,18}$, assim como ferramentas propostas na consulta global para tanto, como a Matriz de Análise de Consistência de Valores Estruturantes e de Implementação dos ODS $^{19}$. A abordagem da governança e as tecnologias de gestão estratégica e avaliação apresentadas são produtos da pesquisa "Territórios Sustentáveis, Promoção da Equidade e da Saúde em Comunidades Tradicionais do Mosaico da Bocaina"20.

\section{Agenda pós-2015}

A transição do modelo econômico centrado no Estado de Bem-estar Social para um modelo de Estado neoliberal, disseminada nos anos 1970 e 1980, reduziu os sistemas de proteção social e atribuiu ao mercado a responsabilidade pelo atendimento das necessidades sociais em detrimento do papel dos estados nacionais, redundando em uma deterioração da qualidade de vida das populações $^{11}$. Na década de 2000, quando começaram a ser retomados mecanismos de regulação econômica e proteção social pelos países, a agen$\mathrm{da}$ dos organismos internacionais desencadeou um "ciclo social" que permitiu que a saúde tivesse papel proeminente, o que se refletiu na definição de três entre oito Objetivos de Desenvolvimento do Milênio (ODM) $)^{21-23}$. Simultaneamente, o tema do desenvolvimento sustentável ascendeu com as evidências de desequilíbrio ecológico e a ampliação das desigualdades sociais, apontando a insustentabilidade do modo de produção e consumo hegemônico, que ameaça a existência da vida na Terra. Isto redundou em um conjunto de iniciativas da ONU, como as Conferências de Estocolmo (1972), Rio de Janeiro-Eco 92 (1992), Johannesburg (2002) e a Rio+20 (2012), assim como da sociedade civil, Fórum Global (1992), Fórum Social Mundial (2001), Cúpula dos Povos (2012), que consolidaram a agenda da sustentabilidade globalmente. Denominada Agenda pós2015, e expressa em ODS, essa nova agenda aspira transcender a dos ODM, a partir do aprendizado de seu processo de implementação, destacando-se as dimensões da intersetorialidade, da interescalaridade e da governança expressas no território.

\section{Avaliação dos ODM e transição para os ODS}

Os documentos das Nações Unidas destacam que o processo gerado pelos Objetivos de Desenvolvimento do Milênio foi bem sucedido, atribuindo isso à combinação de crescimento econômico, melhores políticas e compromisso global com os $\mathrm{ODM}^{1,9,10,24,25}$, apontando a rápida redução da pobreza extrema, embora ainda exista "half a billion fewer people living below an international poverty line of $\$ 1.25$ a day. Child death rates have fallen by more than $30 \%$, with about three million children's lives saved each year compared to 2000. Deaths from malaria have fallen by one quarter"9. Defendem que a aprendizagem com os ODM e as decisões da Rio+20 convirjam para a definição de ODS, que dêem continuidade ao melhor "...of the MDGs, with a practical focus on things like poverty, hunger, water, sanitation, education and healthcare" , indo além dos seus limites: atenção insuficiente às populações mais excluídas; não inclusão da violência produzida pelo desenvolvimento; destaque insuficiente à boa governança e a governos e instituições transparentes e democráticas; pouca importância ao crescimento inclusivo; e baixa matricialidade ${ }^{9}$. Enfatizam que a principal limitação dos ODM foi estrutural. MDGs fell short by not integrating the economic, social, and environmental aspects of sustainable development as envisaged in the Millennium Declaration, and by not addressing the need to promote sustainable patterns of consumption and production. The result was that environment and development were never properly brought together ${ }^{9}$. 


\section{Estratégias, Objetivos e Metas}

Para responder a este contexto, o documento do HLP propôs cinco estratégias alinhadas à visão que advoga: a) Leave no one - Garantir que ninguém tenha seus direitos humanos universais negados, bem como suas "basic economic opportunities"; b) Put sustainable development at the core - integrar os três pilares do desenvolvimento sustentável, promovendo inclusão social e suspendendo o ritmo da mudança climática e da degradação ambiental; c) Transform economies for jobs and inclusive growth - uma inflexão "...to sustainable patterns of consumption and production to ensure good job possibilities while moving to the sustainable patterns of work and life that will be necessary in a world of limited natural resources"; d) Build peace and effective, open and accountable institutions for all - construir sociedades pacíficas baseadas nos direitos humanos fundamentais; e) Forge a new global partnership - promover parcerias baseadas em nossa humanidade comum, capaz de promover solidariedade, cooperação e responsabilização conjunta ${ }^{9}$. Destaca a importância de dar concretude às cinco estratégias, e propõe um conjunto de objetivos e metas que, a seu entender, serão capazes de direcionar e avaliar a Agenda pós-2015, reforçando a importância dos indicadores serem "...disaggregated to ensure no one is left behind and targets should only be considered 'achieved' if they are met for all relevant income and social groups".

\section{Objetivos de Desenvolvimento Sustentável}

Considerando os novos desafios apontados, o documento do HLP ressalta que "Goals have shown their value in focusing global eforts, building momentum and developing a sense of global jeopardy. They can be instrumental in crystallising consensus and defining international norms"9. Recomenda um número limitado de objetivos e metas, que devem ser “...SMART: specific, measurable, attainable, relevant and time-bound". Sugere que sejam: estruturalmente impactantes no desenvolvimento sustentável; atendam à demanda social - especialmente de crianças, jovens, mulheres e grupos excluídos; sejam mobilizadores e fáceis de comunicar; flexíveis e aplicáveis a distintas situações locais; baseados em consensos e em acordos já construídos; e "measurable, using credible and internationally comparable indicators, metrics and data, and subject to monitoring". Aponta os riscos de se construir uma agenda com excessivas priorida- des, mais compromissos que decisões, com pouco foco ou focalizada no passado, vertical e não matricial, uma utopia inalcançável ou insuficientemente tensionadora e transformadora. Adota as definições de objetivos, metas e indicadores: "goal - expresse an ambitious, but specific, commitment. Always starts with a verb/action. Example from MDGs: Reduce child mortality. Targets - quantified sub-components that will contribute in a major way to achievement of goal. Should be an outcome variable. Example from MDGs: Reduce by two-thirds, between 1990 and 2015, the under-five mortality rate. Indicators - precise metric from identified detabases to assess if target is being met (often multiple indicators are used). Example from MDGs: Under-5 mortality rate; Infant mortality rate; Proportion of 1 -year olds immunized against measles" 9 . $\mathrm{O} o b$ jetivo deve ser especificamente relacionado a um tema, focalizado em questões de grande impacto sobre o desenvolvimento sustentável, “...measured in terms of the number of people affected, the contribution to social inclusion, and the need to move towards sustainable consumption and production patterns"9. As metas devem ser capazes de materializar a ambição dos objetivos. Podem ser resultados individuais ou de territórios (comunidades, regiões, países, cidades). Devem sempre ser mensuráveis, ainda que se reconheça a necessidade de maior desenvolvimento técnico para desenvolver indicadores apropriados. As metas nacionais refletirão a especificidade e a velocidade de cada local, mas “...in some cases, there may be a case for having a global minimum standard for a target, where the international community commits itself to do everything possible to help a country reach a threshold level. That applies to the eradication of extreme poverty by 2030 , for example" 9 . Isto pode ser estendido a outras áreas, como educação, saúde, alimentação, energia, discriminação de gênero ou acesso à justiça. As únicas metas globais mantidas no documento são as que já haviam sido pactuadas pelo Secretariado-Geral da ONU na Iniciativa de Energia Sustentável para Todos e algumas que só poderiam ser objeto de uma ação global, como é o caso da reforma do sistema financeiro e comercial internacional. Propõe que alguns temas sejam matriciais e estejam incluídos em todos os ODS: paz, iniquidade, mudança climática, cidades, questões inerentes aos jovens, mulheres e padrões de produção e consumo. Eventualmente estes temas também são contemplados em um ODS específico, como paz e mulheres ${ }^{9}$. Ao descrever o que entende por Sustainable consump- 
tion and production patterns, destacado aqui por seu caráter estruturante, admite que o foco principal voltou-se para água, energia e alimentação, e que é importante que seja ampliado. Afirma que as transformações mais significativas serão conduzidas “...by technology, by innovations in product design, by detailed policy guidelines, by education and changed behavior, and by social innovations embedded in communities" " Considera que a maior parte das novas tecnologias e produtos será originária do empresariado e considera positiva a contribuição deste setor ao desenvolvimento sustentável. Por outro lado, aponta que esta contribuição "...must include a willingness, on the part of all large corporations as well as governments, to report on their social and environmental impact, in addition to releasing financial accounts" 9 . Considerando as questões anteriores, o HLP propôs doze Objetivos de Desenvolvimento Sustentável: ODS1. End poverty, ODS 2. Empower girls and women and achieve gender equality, ODS3. Provide quality education and lifelong learning, ODS4. Ensure healthy lives, ODS5. Ensure food security and good nutrition, ODS6. Achieve universal access to water and sanitation, ODS7. Secure sustainable energy, ODS8. Create jobs, sutainable livelihoods, and equitable growth, ODS9. Manege natural resource assets sustainably, ODS10. Ensure good governance and effective institutions, ODS11. Ensure stable and peaceful societies, ODS12. Create a global enabling environment and catalyse long-term finance ${ }^{9}$, que após a análise do OWG tornaram-se dezessete. O OWG assumiu os pressupostos acordados no HLP, mas procurou avançar em aspectos relacionados à governança e na inclusão de objetivos relacionados a temas que antes haviam sido propostos como transversais.

Isso resultou no desdobramento de alguns dos objetivos do HLP em mais objetivos e na criação de novos ODS, relacionados a temas que foram destaque no processo de definição da Agenda.

O ODS9. Manage Natural Resource Assets Sustainably foi desdobrado em três: ODS13. Promote actions at all levels to address climate change, ODS14. Attain conservation and sustainable use of marine resources, oceans and seas e ODS15. Protect and restore terrestrial ecosystems and halt all biodiversity loss.

Foram criados os: ODS10. Reduce inequality within and among countries, ODS11. Build inclusive, safe and sustainable cities and human settlements e ODS12. Promote sustainable consumption and production patterns.
Adicionalmente, o ODS17. Strengthen and enhance the means of implementation and global partnership for sustainable development foi decupado em metas específicas para cada um dos outros dezessseis ODS. O Quadro 1 apresenta os objetivos para a Agenda pós-2015.

\section{Discussão}

\section{A Agenda dos Territórios Sustentáveis e Saudáveis}

Como dito anteriormente, este cenário abre possibilidades para implantação de uma agenda contra-hegemônica que busque a mudança do modo de produção e consumo predominante, porém também permite a atualização do capitalismo como modo de produção hegemônico ${ }^{11}$. Isso implica em um desafio teórico-prático cuja factibilidade e viabilidade precisam ser demonstradas a partir de experiências concretas, demandando a implantação, gestão e avaliação de agendas territorializadas.

Neste sentido, estas devem apoiar a promoção da inclusão social e do desenvolvimento humano sustentável, visando ampliar o acesso à cidadania, a preservação do meio ambiente, o desenvolvimento econômico e a qualidade de vida, tendo a governança intersetorial e participativa como modo de gestão e o território como elemento contextualizador de sua implantação.

\section{Governança, gestão estratégica e avaliação de efetividade}

A factibilidade e viabilidade destas agendas demandam mecanismos de governança e estratégias de gestão que utilizem a intersetorialidade e a interescalaridade como parâmetros de ação territorial e ferramentas de avaliação capazes de produzir evidências de sua efetividade em relação ao desenvolvimento sustentável e saudável.

Para avaliar a consistência da Agenda pós2015, em relação ao desenvolvimento sustentável e saudável, adotou-se a abordagem ecossistêmica e a abordagem comunicativa do Planejamento Estratégico Situacional (PES),

...pois pressupõem a participação social na gestão das politicas, particularmente da comunidade local, a partir da análise situacional e estratégica, produzindo e utilizando informações diversas que possibilitem demonstrar as interfaces entre bens e serviços dos vários ecossistemas. Também buscam estabelecer mecanismos de governança que abran- 
Quadro 1. Proposed Goals on Sustainable Develepment for the Post2015 Development Agenda.

\begin{tabular}{|l|}
\hline 1. End poverty in all its forms everywhere \\
\hline 2. End hunger, achieve food security and adequate nutrition for all, and promote sustainable agriculture \\
\hline 3. Attain healthy life for all at all ages \\
\hline 4. Provide equitable and inclusive quality education and life-long learning opportunities for all \\
\hline 5. Attain gender equality, empower women and girls everywhere \\
\hline 6. Secure water and sanitation for all for a sustainable world \\
\hline 7. Ensure access to affordable, sustainable, and reliable modern energy services for all \\
\hline 8. Promote strong, inclusive and sustainable economic growth and decent work for all \\
\hline 9. Promote sustainable industrialization \\
\hline 10. Reduce inequality within and among countries \\
\hline 11. Build inclusive, safe and sustainable cities and human settlements \\
\hline 12. Promote sustainable consumption and production patterns \\
\hline 13. Promote actions at all levels to address climate change \\
\hline 14. Attain conservation and sustainable use of marine resources, oceans and seas \\
\hline 15. Protect and restore terrestrial ecosystems and halt all biodiversity loss \\
\hline 16. Achieve peaceful and inclusive societies, rule of law, effective and capable institutions \\
\hline 17. Strengthen and enhance the means of implementation and global partnership for sustainable development \\
\hline Fonte: UN. Open Working Group (OWG). Introduction and proposed goals and targets on sustainable development for the \\
post2015 development agenda, $2014^{10}$.
\end{tabular}

jam as políticas ambientais, sociais e econômicas e que resultem em propostas de gestão integrada, a partir da formulação e efetividade de programas, políticas e projetos ${ }^{8}$.

Esta abordagem permite a análise de consistência, por articular os referenciais teóricos críticos adotados à ação concreta, pragmática. Inicialmente, duas categorias permitiram este vínculo teórico-prático: Projeto e Territorialidade.

'Projeto', considerado como "superação subjetiva da objetividade em direção à objetividade, tenso entre as condições objetivas do meio e as estruturas objetivas do campo dos possíveis"26, que busca atualizar-se em organizações enquanto cristalização institucional de uma correlação de forças ${ }^{27}$. 'Territorialidade', como inscrição da práxis humana, expressão, sobre determinado território, das práticas sociais nele desenvolvidas, do poder sobre o espaço ${ }^{28,29}$. Evidentemente, tanto o Projeto como a Territorialidade pressupõem a existência de sujeitos individuais ou coletivos, portadores de projetos e atores sobre o território. Construtores de territorialidades ${ }^{30}$.

Portanto, o Projeto precisa partir da territorialidade e expressar-se nela mesmo para objetivarse. Nesse sentido, sempre será situacional, o que exige a análise do contexto estratégico, da correla- ção de forças sobre o território e das competências necessárias à sua implantação e implementação. Em outras palavras, mecanismos de governança e gestão estratégica que garantam isto.

O primeiro momento de análise de consistência da Agenda pós-2015 consistiu em verificar se ela atende a essas exigências, utilizando o Triângulo de Governo ${ }^{31}$ que, além do Projeto, destaca a Governabilidade e Capacidade de Governo (Figura 1).

“[...] a 'Governabilidade’ refere-se à relação entre variáveis e recursos que o ator controla e aqueles que não controla; 'e a Capacidade de Governo’ é resultante da mobilização adequada de métodos, técnicas, conhecimentos e experiências adequadas à condução do processo social no sentido do Projeto e levando em conta a Governabilidade do sistema. Estas dimensões têm características distintas, mas condicionam-se reciprocamente e são relativas a uma situação específica, não podendo ser inferidas em abstrato" ${ }^{30}$.

O Triângulo de Governo avalia se a agenda territorializada é produto da pactuação dos pressupostos, categorias e de seu significado, que constituem o Projeto enquanto valores e objetivos; da análise dos interesses dos atores e de seu potencial de ação sobre o território, relativo à go- 


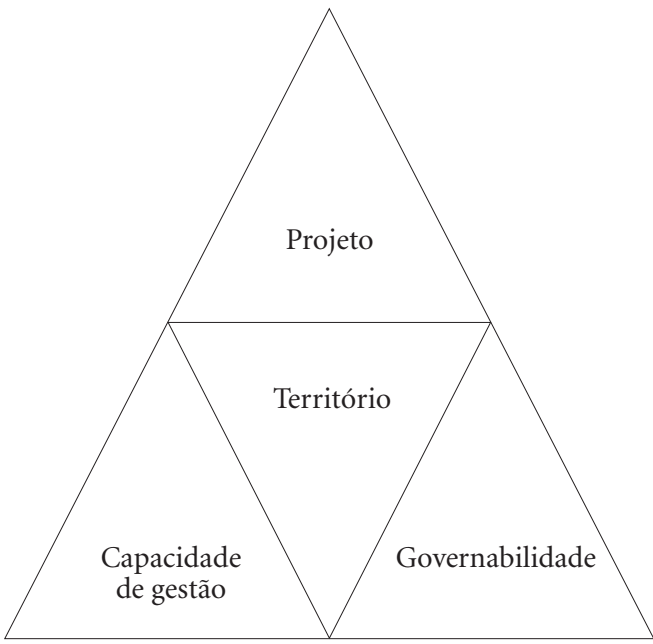

Figura 1. Triângulo de Governo e Território.

vernabilidade; e de seus meios e modo de gestão, que se voltam para a ampliação da Capacidade de Governo como potencial de desenvolvimento das condições de possibilidade de atualização do Projeto $^{30}$.

Esta ferramenta, aplicada à análise da Agenda pós-2015, permite afirmar que esta contempla mecanismos de governança e estratégias de gestão que utilizam a intersetorialidade e a interescalaridade como parâmetros de ação territorial.

Isto é constatado na afirmação de ir além dos limites identificados nos ODM e incluir populações mais excluídas; promover a boa governança; garantir a matricialidade dos ODS; privilegiar o local e avaliar os resultados. Do mesmo modo, na proposição de que sejam estruturalmente impactantes no desenvolvimento sustentável; atendam à demanda social; sejam mobilizadores e fáceis de comunicar; flexíveis e aplicáveis a distintas situações locais; e baseados em consensos e em acordos já construídos?.

Isso também é verificado na definição das características do objetivo, que deve ser relacionado a um tema, focalizado em questões de grande impacto, capazes de promover a mudança para padrões sustentáveis de produção e consumo, e mensurar quantitativamente e qualitativamente a população afetada, considerando especialmente a inclusão social ${ }^{9}$.

Adicionalmente, é proposta uma inovação em relação à governança, que aparece na pac- tuação do ODS10. Reduce inequality within and among countries e no detalhamento do ODS17. Strengthen and enhance the means of implementation and global partnership for sustainable development, com metas específicas para cada um dos outros dezessseis ODS.

Isto visa promover ações interescalares e intersetoriais que possam atender a necessidades dos territórios, bem como pactuar metas que possam ser monitoradas e avaliadas.

Por outro lado, refletindo a disputa de projetos que a contextualiza, a Agenda pós-2015 contém elementos contraditórios que revelam a influência de uma visão liberal e economicista. Isto se reflete, por exemplo, no papel apontado para o setor privado, na promoção da sustentabilidade sem analisar as contradições que lhes são inerentes, por sua inserção no modo de produção e consumo hegemônicos.

Do mesmo modo, a saúde é entendida como elemento de aumento da produção e renda, concebida como "direito humano e universal em sua dimensão básica (acesso universal aos cuidados básicos de saúde); uma mercadoria cujo consumidor - e não o cidadão - deve ser protegido das 'falhas do mercado' (efeitos financeiros catastróficos); e a ênfase seja dada às populações vulneráveis, ainda que se faça referência pontual à promoção da saúde e à sua determinação social e articulação com os pilares do desenvolvimento sustentável [...]". Nessa lógica, apresenta um raciocínio bastante linear, semelhante ao "ciclo virtuoso" desenvolvimentista dos anos 60: "A saúde permite às pessoas atingirem seu potencial. Crianças saudáveis aprendem melhor. Elas se tornarão adultos saudáveis. Adultos saudáveis trabalham mais tempo e mais regularmente, recebendo salários mais altos e regulares" ${ }^{\prime \prime, 17}$.

Apesar disso, o objetivo definido mais diretamente para a saúde, ODS3. Attain healthy life for all at all ages, especialmente se analisadas suas metas, representa um avanço em relação às proposições anteriores, principalmente às da OMS, que defendia a cobertura universal de saúde como um ODS, e no sentido estrito do termo, como apontado acima. Corretamente, esta foi definida como uma meta, 3.6 achieve universal health coverage (UHC), including financial risk protection, with particular attention to the most marginalized and people in vulnerable situations ${ }^{10}$, em um nível mais apropriado.

Para o segundo momento da análise de consistência da Agenda pós-2015, foi utilizada a Matriz de Análise de Efetividade de Estratégias Territorializadas de Desenvolvimento Sustentável e 
Saúde ${ }^{32}$ (Quadro 2), testada anteriormente em análise de consistência e de efetividade de uma agenda territorializada. Para possibilitar a avaliação de efetividade, seguindo o percurso cognitivo do abstrato (essência) ao concreto (experiência), utilizou-se um conjunto de dimensões analíticas e parâmetros que podem constituir índices e indicadores relacionados à interseção entre desenvolvimento, saúde e ambiente.

No que se refere à autonomia, verifica-se que é destacada a importância da participação social, afirmando-se a necessidade de envolvimento dos diversos atores, inclusive do setor privado, bem como a importância do empoderamento social (étnico, de gênero, de grupos excluídos, entre outros) e de modos de governança participativos.

Por outro lado, como apontado no primeiro momento da análise, há uma visão acrítica e limitada do papel do setor privado, ao considerar positiva a contribuição deste setor ao desenvolvimen- to sustentável sem analisar suas contradições. ${ }^{9}$ Os mecanismos de governança, apontados como essenciais para o alcance das mudanças nas formas de produção e consumo e que implicariam em abordagens integradas para as agendas da economia política, da justiça social e ecológica ${ }^{16}$,embora tenham aprofundado a proposição do HLP, correm o risco de se restringir a uma agenda liberal, concentrada em direitos individuais básicos, sem considerar o complexo processo de gestão do desenvolvimento sustentável, o que exigirá o aprofundamento de conceitos, práticas, mecanismos e ferramentas do planejamento e gestão pública intersetorial nas esferas nacionais e global ${ }^{17}$.

No que se refere à equidade, a Agenda considerou a necessidade de promover a inclusão de populações vulneráveis, especialmente de crianças, jovens, mulheres e grupos excluídos. Isto também se reflete no destaque dado à erradicação da pobreza, à afirmação de que os objetivos

Quadro 2. Matriz de análise de efetividade de estratégias territorializadas de desenvolvimento sustentável e saúde.

\begin{tabular}{|c|c|c|c|}
\hline Dimensões Analíticas & Parâmetros Avaliativos & Índices & Indicadores \\
\hline \multirow{8}{*}{ Sustentabilidade } & \multirow{3}{*}{ Intersetorialidade } & Convergência de Agendas & \\
\hline & & Integração de stakeholders & \\
\hline & & Integração interescalar & \\
\hline & Redes de Solidariedade & Inserção em Redes & \\
\hline & \multirow{2}{*}{ Territorialização } & Necessidades do território & \\
\hline & & Hierarquia de prioridades & \\
\hline & \multirow{2}{*}{ Ecologia de saberes } & Espaços coletivos de gestão & \\
\hline & & Mecanismos de produção de conhecimentos & \\
\hline \multirow{8}{*}{ Equidade } & \multirow{3}{*}{ Diversidade } & Raça & \\
\hline & & Gênero & \\
\hline & & Biodiversidade & \\
\hline & \multirow{3}{*}{ Vulnerabilidade } & Renda & \\
\hline & & Ciclo de vida & \\
\hline & & Acesso & \\
\hline & \multirow[b]{2}{*}{ Integralidade } & Acolhimento & \\
\hline & & $\begin{array}{l}\text { Vínculo (tecnologias leves promotoras de } \\
\text { saúde) }\end{array}$ & \\
\hline \multirow{6}{*}{ Autonomia } & \multirow{2}{*}{ Participação social } & Capacidade reivindicativa & \\
\hline & & Intensidade de participação & \\
\hline & \multirow{4}{*}{ Empoderamento } & Capacidade de formulação & \\
\hline & & Coordenação de Redes & \\
\hline & & Capacidade de gestão & \\
\hline & & Resiliência & \\
\hline
\end{tabular}


devem incluir a todos e que somente serão considerados atingidos quando alcançarem todos os segmentos sociais, especialmente os mais excluídos, que buscam materializar a diretriz leave no one behind.

Neste sentido, afirma que "a pobreza tem várias manifestações, incluindo a falta de renda e recursos produtivos suficientes para garantir um nível de vida sustentável, fome e má nutrição, saúde deficiente, acesso limitado ou ausente à educação e outros serviços básicos, grande morbidade e mortalidade devido a doenças, habitação inadequada, ambiente inseguro, discriminação e exclusão social. É também caracterizada por falta de participação no processo decisório, social e cultural"17.

No entanto, o conjunto de metas demonstrou ser insuficiente em medidas econômicas ou de organização social que favoreçam o empoderamento desses grupos ou o próprio fortalecimento do processo democrático. Os esforços para melhorar a renda da mulher, por exemplo, enfocaram exclusivamente a legislação e as políticas sobre trabalho e mercados, sem tratar adequadamente as questões referentes à exposição à violência de gênero, estereótipos culturais e divisões de gênero no trabalho intrafamiliar, bem como as questões mais amplas de política macroeconômica e distribuição de recursos.

Em relação à dimensão da sustentabilidade, há preocupação com modos de governança baseados em instituições transparentes e eficazes, e com a intersetorilidade e interescalaridade. A governança é entendida como fator crítico para a plena realização da Agenda, com destaque à continuidade dos ODM, universalidade dos ODS, adaptabilidade às diferentes esferas políticas, intersetorialidade devido à visão holística do processo de desenvolvimento sustentável, coordenação e coerência entre os diversos setores da esfera pública, participação social e responsabilização dos diversos atores ${ }^{17}$.

Isso pode ser verificado nas proposições relacionadas à criação de um ambiente global favorável ao desenvolvimento sustentável, no reconhecimento das responsabilidades comuns, mas diferenciadas, entre os países com distintos graus de desenvolvimento, que redundou nos ODS10 e ODS17, e nas metas a eles relacionadas.

No entanto, como destacado anteriormente, as causas estruturais dos modos de produção e consumo e da distribuição desigual de poder, que são as produtoras das iniquidades e impedem o verdadeiro desenvolvimento ${ }^{33}$, ainda não foram enfrentadas com a radicalidade necessária, como se observa nas metas dos ODS8, ODS9 e ODS12. As deste último, especialmente, têm um alcance extremamente limitado, desproporcional à ambição do Objetivo.

No terceiro momento da análise de consistência da Agenda pós-2015, foi utilizada a Matriz de Análise de Consistência de Valores Estruturantes e de Implementação dos $\mathrm{ODS}^{19}$, representada no Quadro 3.

Ao analisar-se a Agenda com esta ferramenta, foi possível identificar que os elementos que compõem seu Projeto alinham-se aos valores estruturantes da Matriz 3: Sustentabilidade Ambiental, Erradicação da Pobreza, Direitos $\mathrm{Hu}$ manos, Equidade e Inclusão e Responsabilidade Global; os relacionados à sua Governabilidade aos valores de implementação: Accountability e Participação; e os relativos à sua Capacidade de Governo aos objetivos Baseados em Evidências e à Efetividade, o que reforça os dois primeiros momentos da análise de consistência.

Adicionalmente, a partir da análise de consistência dos dezessete temas propostos para a Agenda pós-2015, nos três momentos anteriores, especialmente no que se refere à e interescalaridade, à intersetorialidade e à governança territorializada, verificou-se que:

- Um é um metaobjetivo, que aponta para um novo modo de organização da produção e consumo e, consequentemente, de arranjo social (ODS12. Promote sustainable consumption and production patterns)

- Oito correspondem aos pilares do desenvolvimento sustentável - econômico: ODS8. Promote strong, inclusive and sustainable economic growth and decent work for all; ODS9. Promote sustainable industrialization ambiental: ODS13. Promote actions at all levels to address climate change; ODS14. Attain conservation and sustainable use of marine resources, oceans and seas; ODS15. Protect and restore terrestrial ecosystems and halt all biodiversity loss; social: ODS1. End poverty in all its forms everywhere; ODS5. Attain gender equality, empower women and girls everywhere; ODS10. Reduce inequality within and among countries

- Cinco são considerados focos de ação (ODS2. End hunger, achieve food security and adequate nutrition for all, and promote sustainable agriculture; ODS3. Attain healthy life for all at all ages; ODS4. Provide equitable and inclusive quality education and life-long learning opportunities for all; ODS6. Secure water and sanitation for all for a sustainable world; ODS7. Ensure access to affordable, sustainable, and reliable modern energy services for all) 
- Dois são variáveis territoriais, que refletem a vulnerabilidade e a diversidade: ODS11. Build inclusive, safe and sustainable cities and human settlements; ODS16. Achieve peaceful and inclusive societies, rule of law, effective and capable institutions
- Um, a Governança, corresponde ao modo de gestão dos ODS - ODS17. Strengthen and enhance the means of implementation and global partnership for sustainable development

A Figura 2 representa a relação entre os pilares do desenvolvimento sustentável, seus focos de ação, sua convergência territorial e governança.

Quadro 3. Matriz de Análise de Consistência de Valores Estruturantes e de Implementação dos ODS.

\begin{tabular}{|l|l|l|l|}
\hline & $\begin{array}{c}\text { Framework Criteria } \\
\text { evaluating overall coherence of } \\
\text { the group of goals selected to } \\
\text { achieve our purpose and secure } \\
\text { progress towards our vision }\end{array}$ & $\begin{array}{c}\text { Goals Criteria } \\
\text { evaluating each goal } \\
\text { proposed }\end{array}$ & $\begin{array}{c}\text { Targets Criteria } \\
\text { evaluating the } \\
\text { targets identified } \\
\text { urder each goal }\end{array}$ \\
\hline Environmental Sustainability & & Foundation Values & \\
\hline Poverty Eradication & & & \\
\hline Well-being & & & \\
\hline Human Rights & & & \\
\hline Equity & & & \\
\hline Global Responsibility & & & \\
\hline Peace and Security & & Implementation Values & \\
\hline Accountability & & & \\
\hline Evidence-Based & & & \\
\hline Effectiveness & & & \\
\hline Participation & & & \\
\hline
\end{tabular}

Fonte: Beyond. Campaigning for a global development framework after the Millenium Development Goals, $2015^{19}$.

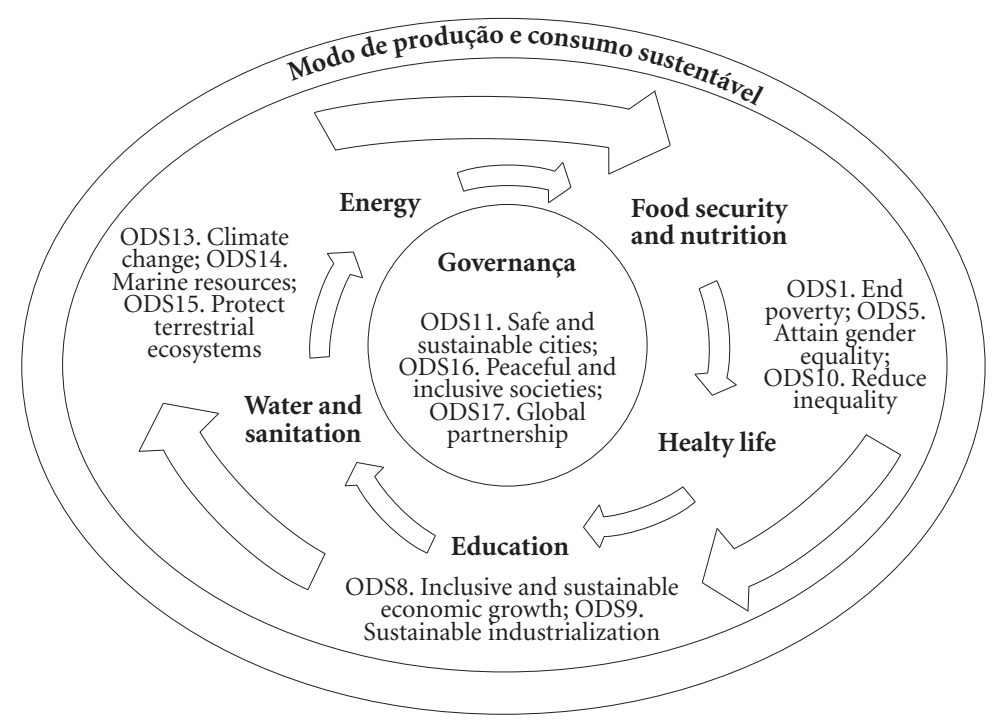

Figura 2. Território, Governança, Interescalaridade e Intersetorialidade na Agenda pós-2015. 
O diagrama demonstra que a Agenda contempla os pilares do desenvolvimento sustentável, o território, a interescalaridade e a intersetorialidade, mas principalmente, aponta o desafio de construção de sua efetividade, que implica no alinhamento estratégico e no desenvolvimento de capacidade de gestão dos planos para os focos de ação, considerando-se as variáveis territoriais que irão determinar a especificidade da sua aplicação local, sua governabilidade.

\section{Conclusão}

Em sua formulação, e provavelmente em sua implementação, a Agenda do Desenvolvimento pós-2015 permite uma tematização estrutural, ao assumir a insustentabilidade do atual modo de produção e consumo. Isto é uma condição de possibilidade para a implantação de um modo alternativo, solidário e cooperativo.

Sua efetividade, entretanto, dependerá de seu rebatimento sobre o território, de sua expressão em agendas territorializadas, cuja governança e gestão estratégica, em especial a avaliação de efetividade são os desafios mais relevantes, demandando objetivos, metas e estratégias matriciais capazes de articular os pilares do desenvolvimento sustentável.

A Agenda atende a estes requisitos em sua formulação, ao afirmar a importância da governança e a intersetorialidade e interescalaridade como elementos críticos.

O destaque dado à governança, associado à perspectiva de atender as necessidades locais articulando-as a parâmetros regionais, nacionais e globais, coloca o território como centro na definição da hierarquia de necessidades e de implementação de soluções, o que é um fator crítico para sua efetividade, embora os conflitos inerentes ao embate entre projetos distintos e mesmo antagônicos, cujos vetores atuam produzindo o espaço e territorialidades, não sejam explorados devidamente.

Atende à interescalaridade, ao afirmar que todos os ODS devem ser universais, mas que quase todas metas deverão ser definidas nacional ou localmente, de forma a atender as singularidades dos territórios. Neste sentido, poucas metas propostas são globais, estabelecendo um parâmetro comum e mensurável a ser monitorado em todos os países, como, p.e., a duplicação da taxa de energia renovável na matriz energética global. Algumas metas podem ser constituídas por parâmetros mínimos, pactuados entre todos os países, como, p.e., a taxa máxima de mortalidade infantil.

Quanto à intersetorialidade, os objetivos pactuados têm nitidamente esta perspectiva, como, p.e., os ODS1. End poverty in all its forms everywhere e ODS16. Achieve peaceful and inclusive societies, rule of law, effective and capable institutions, e há a preocupação com mecanismos de governança integradores, como se verifica no detalhamento das metas do ODS17, com conjuntos específicos para cada um dos outros dezesseis ODS.

Adicionalmente, é destacada a importância de desenvolvimento de sistemas, tecnologias e ferramentas de monitoramento e avaliação, para a construção de indicadores mensuráveis e potentes.

Uma importante justificativa para essa ênfase relaciona-se à equidade, na medida em que as médias escondem as desigualdades e iniquidades, e que quanto mais desagregado o indicador, maior a possibilidade de identificação de tendências e eventos-sentinela. Neste sentido, é recomendada a sua desagregação ...with respect to income (especially for the bottom 20\%), gender, location, age, people living with disabilities, and relevant social group. Targets will only be considered 'achieved' if they are met for all relevant income and social?.

Esta formulação abre possibilidades de promoção de ações interescalares e intersetoriais que possam atender às necessidades dos territórios, bem como pactuar metas e indicadores que possam ser monitorados e avaliados.

No entanto, contextualizada pela disputa de projetos históricos de sociedade, a análise situacional apresentada na Agenda pós-2015, ainda que reconhecendo a inviabilidade do modo de produção e consumo capitalista - expressão não utilizada em quaisquer dos documentos - elude suas causas estruturais, deixando de reconhecer sua persistência "genética" nos sujeitos e nas estratégias que promovem. Isso resulta em uma visão muitas vezes ingênua, como se os atores capitalistas, em conjunto, de repente passassem a conduzir seus planos estratégicos abandonando o telos do lucro e os mecanismos de exploração da força de trabalho em nome da sustentabilidade e da solidariedade. O mesmo é válido para a posição dos Estados nacionais, eludindo seus anéis burocráticos com o capital.

No que se refere à capacidade da abordagem e das ferramentas avaliativas propostas na análise de consistência da formulação da Agenda, podese afirmar que a cartografia resultante do procedimento analítico adotado permitiu um olhar panorâmico sobre a Agenda pós-2015, a análise 
de seu contexto estratégico, correlação de forças e competências necessárias à sua implantação e implementação, utilizando o Triângulo de Governo, assim como seu grau de alinhamento às dimensões estabelecidas na Matriz de Análise de Efetividade de Estratégias Territorializadas de Desenvolvimento Sustentável e Saúde e na Matriz de Análise de Consistência de Valores Estruturantes e de Implementação dos ODS.

Considera-se que a abordagem e as tecnologias utilizadas têm potencialidade explicativa para análise de consistência da formulação de Projetos, e permitem um aprofundamento de análises particulares de cada uma das dimensões e parâmetros utilizados, realimentando o processo de gestão das agendas territorializadas, fornecendo subsídios para a tomada de decisão e para a formulação de políticas, programas e projetos que promovam territórios sustentáveis e saudáveis, assim como podem vir a se constituir em ferramenta de apoio ao monitoramento e avaliação dos Objetivos de Desenvolvimento Sustentável - ODS.

Finalmente, avalia-se que a formulação da Agenda pós-2015 representa um avanço em relação à Agenda do Milênio, e que sua implantação delimita um contexto que abre possibilidades para a definição de uma agenda de implantação de um novo modo de produção e organização social, mais cooperativo e solidário, capaz de promover a justiça socioambiental, ainda que isto dependa dos processos históricos de disputa de hegemonia, o que demanda a necessidade de articulação dos diferentes atores e redes sociais que compartilham da mesma visão de mundo para a construção de uma Agenda contra-hegemônica de implementação dos ODS e de mecanismos de governança capazes de implementá-los territorialmente.

\section{Colaboradores}

E Gallo e AFF Setti participaram igualmente de todas as etapas de elaboração do artigo. 


\section{Referências}

1. United Nations (UN). El futuro que queremos, 2012. [acessado 2013 mar 29]. Disponível em: http://www. uncsd2012.org/content/documents/778futurewewant _spanish.pdf

2. International Conference on Urban Health (ICUH). Final Report 10th International Conference on Urban Health. Minas Gerais, 2011. [acessado 2014 jun 14]. Disponível em: http://issuu.com/osubh/docs/icuh2011 _report?e $=6544358 / 2806754$

3. Programa das Nações Unidas para o Desenvolvimento (PNUD). Combater as alterações climáticas: solidariedade humana num mundo dividido. Relatório de Desenvolvimento Humano 2007/2008. [acessado 2008 jun 01]. Disponível em: $<$ http://www.pnud.org.br/arquivos /rdh/rdh20072008/hdr_20072008_pt_complete.pdf

4. Comissão Nacional sobre Detereminantes Sociais da Saúde (CNDSS). As Causas Sociais das Iniquidades em Saúde no Brasil - Relatório Final, 2008. [acessado 2011 mar 03]. Disponível em: http://www.cndss.fiocruz.br/ $\mathrm{pdf} /$ home/relatorio.pdf

5. United Nations Conference on Trade and Development (UNCTAD). The Road to Rio+20 - For a developmentled green economy. New York, Geneva: UNCTAD; 2011.

6. United Nations Environment Panel (UNEP). Towards a Green Economy: Pathways to Sustainable Development and Poverty Eradication - A Synthesis for Policy Makers, 2011. [acessado $2011 \mathrm{fev}$ 13]. Disponível em: www. unep.org/greeneconomy.

7. United Nations (UN). United Nations Millennium Declaration. [acessado 2011 maio 14]. Disponível em: www.un.org/millenniumgoals/bkgd.shtml

8. Gallo E, Setti AFF. Abordagem ecossistêmica e comunicativa na implantação de Agendas territorializadas de desenvolvimento sustentável e promoção da saúde. Cien Saude Colet 2012; 17(6):1433-1446.

9. United Nations (UN). The Report of the High-Level Panel of Eminent Persons on the Post-2015 Development Agenda: A New Global Partnership - Eradicate Poverty and Transform Economies through Sustainable Development. [acessado 2014 mar 21] Disponível em: http:// www.post2015hlp.org/wp-content/uploads/2013/05/ UN-Report.pdf

10. United Nations (UN). Open Working Group (OWG). Introduction and proposed goals and targets on sustainable development for the post2015 development agenda. [acessado 2014 jun 25]. Disponível em: http:// sustainabledevelopment.un.org/content/documents /4044140602workingdocument.pdf

11. Gallo E, Setti AFF, Magalhães DP, Machado JMH, Buss DF, Netto FAF, Buss PM. Saúde e economia verde: desafios para o desenvolvimento sustentável e erradicação da pobreza. Cien Saude Colet 2012; 17(6):1457-1468.

12. United Nations (UN). Communique on the Secretary General's High Level Panel of Eminent Persons on the Post-2015 Development Agenda, 2012. [acessado 2013 mar 29]. Disponível em: http://www.worldwewant 2015.org/bitcache/15d41549a964b69d1dbee3c7926e $4920 \mathrm{e} 9 \mathrm{cb} 3 \mathrm{af} 7$ ?vid $=307681 \&$ disposition $=$ attachment \&op=download

13. Gallo E, Setti AFF. Efetividade em desenvolvimento sustentável: o caso do Projeto Bocaina. In: Anais do GeoSaude 2014: A geografia da saúde no cruzamento de saberes; 2014; Coimbra. p. 696-699.
14. Comissão Nacional sobre Determinantes Sociais daSaúde no Brasil (CNDSS). As Causas Sociais das Iniqüidades em Saúde no Brasil - Relatório Final, 2008. Pár. 15.2 (iii). [acessado 2011 mar 03]. Disponível em: http://www.cndss.fiocruz.br/pdf/home/relatorio.pdf

15. United Nations (UN). Dialogue on Health in Post-2015 Development Agenda Gabarone, 4-6 March 2013: Meeting report. [acessado 2014 jun 15]. Disponível em: http://globalhealth.ie/uploads/files/Meeting\%20Report \%20Botswana_03-2013.pdf

16. United Nations (UN). Thematic Consultations. [acessado 2014 jun 15]. Disponível em: http://www.world wewant2015.org/sitemap\#thematic

17. Buss PM, Pellegrini Filho A, Matida A, Setti AFF, Buss DF, Magalhães D, Gallo E, Netto FF, Netto GF, Machado JH. Saúde na Agenda do Desenvolvimento pós-2015. Informe preliminar do Grupo Técnico Fiocruz sobre Desenvolvimento Sustentável e Saúde. Rio de Janeiro: Fundação Oswaldo Cruz (Fiocruz); 2013.

18. Gallo E, Setti AFF. Desenvolvimento Sustentável e Promoção da Saúde: implantação de agendas territorializadas e produção de autonomia. Saude Deb 2012; 36(N. esp.):55-67.

19. Beyond 2015. Campaigning for a global development framework after the Millenium Development Goals. [acessado 2014 maio 23]. Disponível em: http://www. beyond2015.org/document/criteria

20. Fundação Oswaldo Cruz (Fiocruz) - Vice-Presidência de Ambiente, Atenção e Promoção da Saúde (VPAAPS) e Fundação Nacional de Saúde (FUNASA) - Departamento de Saúde Ambiental (Desam). Projeto de Pesquisa TC 01/2013. Territórios Sustentáveis, Promoção da Equidade e da Saúde em Comunidades Tradicionais do Mosaico da Bocaina, 2013.

21. Belinky A. Uma economia democrática para o desenvolvimento sustentável. 2012. [acessado 2012 abr 17]. Disponível em: http://rio20.net/pt-br/documentos/uma -economia-democratica-para-o-desenvolvimentosustentavel

22. Pietrovsky I. Por trás do documento que pautará a Rio+20 oficial. 2012. [acessado 2012 abr 17]. Disponível em: http://rio20.net/pt-br/documentos/por-trasdo-documento-que-pautara-a-rio20-oficial

23. The Lancet. Global health in 2012: development to sustainability. The Lancet 2012; 379(9812):193.

24. United Nations (UN). Department of Economic and Social Affairs and the UN Department of Public Information DPI/2363/Rev.2. September, 2005. [acessado 2014 maio 23]. Disponível em: http://www.un.org/ milleniumgoals/pdf/mdg2005progresschart.pdf

25. United Nations (UN). The Millennium Development Goals Report, 2010. [acessado 2014 maio 23]. Disponível em: http://un.org/millenniumgoals/pdf/MDG\%20 Report\%202010\%20En\%20r15\%20-low\%20res\%20 20100615\%20-.pdf

26. Sartre JP. Questão de método. In: Sartre JP. Os Pensadores. São Paulo: Abril Cultural; 1973. p.160.

27. Poulantzas N. O estado, o poder, o socialismo. Rio de Janeiro: Graal; 1981

28. Lèfebvre H. O direito à cidade. São Paulo: Moraes; 1991.

29. Harvey D. Condição pós-moderna. São Paulo: Loyola; 1993. 
30. Gallo E, Freitas LE, Reis R. Flexibilidade, Responsabilização e Autonomia: o caso da Diretoria de Investimentos e Projetos Estratégicos do Ministério da Saúde (DIPE - MS). Saude Deb 2006; 30:58-79.

31. Matus C. Política, planejamento e governo. Brasília: IPEA; 1993.

32. Gallo E, Setti AFF. Efetividade em desenvolvimento sustentável: Desenvolvimento de tecnologias de avaliação. In: Anais do GeoSaude 2014: A geografia da saúde no cruzamento de saberes; 2014; Coimbra. p. 709-713.

33. Buss PM, JMH Machado, E Gallo, DP Magalhães, AFF Setti, FA Franco Netto, DF Buss. Governança em saúde e ambiente para o desenvolvimento sustentável. Cien Saude Colet 2012; 17(6):1479-1491.

Artigo apresentado em 30/06/2014

Aprovado em 15/08/2014

Versão final apresentada em 16/08/2014 